Brit. J. Psychiat. (1970), $117,117-128$

\title{
Correspondence
}

\section{TREATMENT OF PHOBIC PATIENTS WITH ANTIDEPRESSANTS}

\section{Dear Sir,}

Your April number carries a report (I) on a retrospective study of 246 phobic patients treated with M.A.O.I. or 'combined' antidepressants. The patients were treated, and the study conducted and written up, by psychiatrists on the staff of one of London's most distinguished teaching hospitals; the authors used the word 'significant' (variously qualified as 'statistically' or 'highly') no less than eighteen times in relation to the positive results being reported; and the paper received the imprimatur of publication in British psychiatry's most respected journal. It seems likely that the authors' 'finding' that tratement with an M.A.O.I., alone or in combination, 'results in' a 'significant' improvement in patients who have phobias as their main symptoms will receive wide acceptance. This, I suggest, would be unfortunate, since whether or not M.A.O.I. drugs are in fact effective in the treatment of phobias their effectiveness is certainly not established by the study under discussion.

1. The study was uncontrolled; as long ago as 1957 Foulds (2) reported in the Journal of Mental Science that authors claimed success in psychiatric treatment in 85 per cent of uncontrolled studies compared to 19 per cent of studies in which controls were used; apparently the moral has yet to be learnt.

2. There were no data which were not unavoidably bias-prone by virtue of their initial source, i.e. the clinical records of the doctors responsible for prescribing the treatment.

3. There was no standardization of the method of data collection; instead rating was carried out subsequently on the clinical records and this inevitably somewhat arbitary retrospective method of quantification was used to yield numerical values of dubious validity which were then subjected to statistical manipulations of doubtful appropriateness to figures so derived.

4. No attempt was made to avoid compounding the error associated with point ' 2 ' by having the subsequent rating of the clinical records performed blind.

5. No attempt was made to investigate the degree of unreliability stemming from point ' 3 '; even if all the pitfalls previously enumerated were deemed unavoidable it would still have been possible to have each clinical record rated independently by two raters and the inter-rater reliability statistically assessed and stated in the report. Had this been done, the reader would have at least a partial measure of the value of the raw data used in the subsequent statistical manipulations.

6. Tests of statistical significance were applied in gay abandonment of the principle that they are appropriate only where the treatment under investigation and chance are the sole factors operating (3) (or, if other factors might be operating, when either they have been controlled for, or their effects have been isolated and themselves examined statistically). Before tests of statistical significance can be taken to signify anything more than the authors' pretensions it is absolutely essential that factors such as the passage of time, suggestion, placebo reaction and bias should be eliminated by the basic procedures of employing controls, random allocation between groups and blind ratings. 'The application of statistical significance tests to trials not conducted thus is inappropriate ... especially ... when patients treated recently are compared with those treated previously (retrospective comparison). In such cases it cannot be assumed that the groups are comparable'(3). In fact, Dr. Kelly and his colleagues actually applied tests of significance to comparisons with a group of patients treated elsewhere by (an outmoded method of) behaviour therapy, and attach some importance to the result of their comparison, despite admitting that the groups are not really comparable in that the majority of the behaviour therapy patients had more severe illnesses of longer duration than the authors' own patient group.

'There are some people who try to stifle disagreement by proclaiming that their results are "statistically significant", as though that had finality or even proved somehow that the design of the experiment was irreproachable-than which nothing could be further from the truth'(3). So it would seem.

Certain of the above issues were, in fact, touched on in the discussion section of the report, perhaps with a view to forestalling criticism of the manner in which they were disregarded in the actual conduct of the investigation.

The authors suggest that 'spontaneous recovery could probably be ruled out with very many of' (their adult patients) because of the long mean duration of symptoms; in the majority of their cases, however, it is 'improvement' and not recovery which 
is at issue. Phobic patients (especially agoraphobics) are liable to spontaneous fluctuation in the severity of their disability and naturally tend to present and start treatment during a phase of relatively severe affliction. It follows that some degree of improvement can be reasonably expected during the period following presentation in many cases. The uncertainty, even in the authors' minds, implicit in the words 'probably' and 'very many' could be resolved, and the magnitude of degree of spontaneous improvement to be expected in any period following presentation measured and taken into account, by-and only by-having a control group.

Furthermore, it is not, in fact, even the difference between the effect of a specific treatment and the degree of spontaneous improvement over the period following presentation that is at issue: it is rather the effect of a specific treatment versus the summed effects of attention and placebo reaction. The authors seek to reassure themselves and (hopefully) their readers, by mentioning that many patients. had had a great variety of treatments before referral. Their contention that the failure of previous treatments indicates that the effects of 'suggestion' or 'the confidence of the doctor' could be more or less discounted is made less convincing when in the same paragraph they contrast their own manner of prescribing anti-depressants ('giving them with confidence') with the unhelpful (but understandable?) anxiety which they say characterizes 'some doctors' when prescribing M.A.O.Is. And their assertion that the latent interval between starting treatment and the occurrence of improvement was 'solid evidence that the improvement seen was due to the specific effect of the anti-depressants' would be more acceptable if one could be certain that patients were never warned to expect such an interval, and that the regular recording of such a latent interval was in no degree consequent upon the expectations of the prescribing doctors.

Dr. Kelly and his colleagues suggest that 'to carry out a trial using a placebo appears unjustifiable in view of the prolonged length of treatment..., ' but later they go on to state that 'anti-depressants should generally (be tried) because, when successful, the initial response is quicker than with any other type of treatment available at present'. In fact, given that according to the report 'the change in mean phobic ratings at one month ... showed a highly significant improvement', and even allowing for the reported latent interval between stopping treatment and relapse, it would be possible to assess the claimed efficacy of M.A.O.I.s adequately and with suitable rigour in a simple sixteen week doubleblind crossover trial employing a placebo. It is profoundly to be hoped that Dr. Kelly and his associates will now undertake such a trial.

The last decade has seen a proliferation of Academic Departments of Psychiatry within Britain, and we are looking forward hopefully to the establishment of psychiatry's own Royal College: these developments represent not only the aspirations of British psychiatrists, but also the increasing acceptance by our general medical colleagues of the legitimacy of such aspirations. The execution, reporting and publication of investigations the gross methodological inadequacies of which would be apparent to any final year medical student, cannot but imperil this increasing acceptance. Might I humbly suggest, Sir, that the Fournal could appropriately celebrate this auspicious year by a firm policy decision that papers combining those pretensions to scientific respectability which a heavy sprinkling of Probability Values always represents with serious and fundamental flaws in experimental design, will have to look elsewhere for publication?

A. B. Mawson.

The Maudsley Hospital, S.E.5

\section{REFERENCES}

I. Kelly, D. et. al. (1970) 'Treatment of phobic states with antidepressants. Brit. F. Psyshiat. 116, 38798.

2. Foulds, G. A. (1958). 'Clinical research in psychiatry.' 7. ment. Sci., 104, 259.

3. Laurence, D. R. (1962). Clinical Pharmacology London: Churchill.

DeAr Sir,

Dr. Mawson appears to make two main points in his letter: first, the weaknesses inherent in our study because of its retrospective nature, and, secondly, its lack of controls. The evaluation of treatment in psychiatry is a slow and complicated process, especially where drugs are concerned. Initially it is necessary to have considerable experience with a drug, to discover its properties and side-effects, the type of patient who would benefit from treatment, and to form hypotheses about its clinical, as opposed to its pharmacological, mode of action. The next step is to test these hypotheses and to decide whether it would be of value to carry out a prospective controlled trial with placebo, and if this were so how it should best be carried out. A retrospective study, which enables large numbers of patients to be examined, is appropriate in this case, in spite of the potential shortcomings, of which we are aware. In our study a control group was not available 